\title{
Reply to: Effect of axial length on myopic choroidal neovascularization
}

\author{
Jiang-Hui Wang • Wei Jiang $•$ Ze-Feng Kang
}

Received: 17 December 2012 / Accepted: 18 December 2012 /Published online: 5 January 2013

(C) Springer-Verlag Berlin Heidelberg 2013

\section{Dear Editor:}

We thank Zhang et al. for their interest in our recent published paper.

We are aware of paucity of data with conflicting results pertaining to the relation between degree of myopia (refractive error, axial length) and myopic choroidal neovascularization (CNV). In theory, eyes with more severe myopia have better prognosis, likely reflecting greater chorioretinal degeneration and lower vascular endothelial growth factor (VEGF) expression that culminates in less growth of CNV [1]. In addition, it is suggested that more favorable visual results occur in eyes with a higher refractive error than in those with a lower refractive error [2], while other investigators have failed to replicate this finding $[3,4]$. When degree of myopia relates to the effects of intravitreal bevacizumab (IVB) injection due to myopic CNV, it is more complicated when explained explicitly because of the synergistic effect of several factors such as age onset, baseline visual acuity, CNV size and chorioretinal atrophy.

In our study, we searched several databases which resulted in 16 articles according to our search strategy, which mainly screened those randomized, or observational studies. Although there were only three articles with regard to the relation between degree of myopia and best-corrected visual acuity (BCVA) after IVB injection, which also showed the sparse available studies to assess their relation, it still indicated that refractive error and axial length has no

\section{J.-H. Wang}

Department of Ophthalmology and Visual Sciences,

The Chinese University of Hong Kong,

4/F, 147K Argyle Street,

Kowloon, Hong Kong

e-mail: jianghui.wang@cuhk.edu.hk

\section{W. Jiang $\cdot$ Z.-F. Kang $(\bowtie)$}

Eye Hospital of China Academy of Chinese Medical Sciences, \#33 Lugu Road,

Shijingshan District, Beijing 100040, China

e-mail: zefeng2531@163.com significant effect on the BCVA after IVB injection. The result was in line with other therapeutic method such as photodynamic treatment [5]. Moreover, the change and effect of axial length is quite persistent and slow which could not be taken into account in the effect of IVB injection in a short time. From this point of view, we believe that axial relation to BCVA after IVB injection is not significant.

In their letter, Zhang et al. reported that variant inclusion criteria, methodology for $\mathrm{CNV}$ identification/progression and cut-off among studies might affect the efficacy of IVB injection. We agree that the variance such as baseline data, study duration, and IVB protocol, might influence on the inclusion in each study. To date, we still do not understand the pathogenesis of myopic CNV clearly and the nature of association between degree of high myopia and myopic CNV remains unknown. Thus, prospective studies should be conducted to provide insight into the relationship between degree of high myopia and CNV that could shed some light on the link between degree of high myopia and the efficacy of IVB.

\section{References}

1. Kwak N, Okamoto N, Wood JM (2000) VEGF is major stimulator in model of choroidal neovascularization. Invest Ophthalmol Vis Sci 41:3158-3164

2. Pece A, Isola V, Vadalà M, Matranga D (2006) Photodynamic therapy with verteporfin subfoveal for choroidal neovascularization secondary to pathologic myopia: long-term study. Retina 26:746751

3. Maár N, Ergun E, Luksch A, Stur M (2006) Treatment frequency and visual outcome in subfoveal choroidal neovascularization related to pathologic myopia treated with photodynamic therapy. Graefes Arch Clin Exp Ophthalmol 244:1262-1266

4. Montero JA, Ruiz-Moreno JM (2003) Verteporfin photodynamic therapy in highly myopic subfoveal choroidal neovascularisation. Br J Ophthalmol 87:173-176

5. Stur M, Ergun E, Heinzl H (2004) Prognostic visual outcome of photodynamic therapy for subfoveal choroidal neovascularization in pathologic myopia. Am J Ophthalmol 138:434-438 